\title{
A Real-time Framework for Patient Monitoring Systems based on a Wireless Body Area Network
}

\author{
Nora Mahmoud \\ Machine learning and information \\ retrieval Department, Faculty of \\ artificial intelligence, Kafrelsheiksh \\ university, Egypt
}

\author{
Shaker El-Sappagh \\ Centro Singular de Investigación \\ en Tecnoloxías Intelixentes, \\ Universidade de Santiago de \\ Compostela, Santiago, Spain
}

\author{
Hazem M. El-Bakry \\ Information Systems Department \\ Faculty of Computers and Information \\ Mansoura University, Egypt
}

\author{
Samir M. Abdelrazek \\ Information Systems Department, \\ Faculty of Computers and \\ Information, Mansoura University, \\ Egypt
}

\begin{abstract}
Chronic disease is a persistent clinical condition that causes significant limitation in a patient's life, due to ill-health and degradation events which may happen frequently. Therefore, it requires continuous collaborations *between patient and physician in an integrated health care system. Current technologies provide an effective and efficient way of patient monitoring. However, none of the current solutions provide end-to-end systems that covers data extraction, transmission, analysis, storage and integration. In our proposed framework, we aim to fill the gap between current technologies and healthcare systems. The wireless body area network (WBAN), cloud computing, fog computing, semantic ontology, and clinical decision support system (CDSS) are integrated to provide a comprehensive and complete model. By monitoring a person with chronic diseases in real time, physicians will have the ability to guide patients with the right decisions. In addition, patients can practice normal life. Finally, there is no need for the patient to stay at hospitals, which saves money. We will provide a further step by providing an intelligent CDSS capability at the hospital's and the patient's sides. The data collected from WBAN will integrated into the electronic health record (EHR) of the patient, handled in terms of semantic interoperability challenge. The proposed novel framework is feasible, and we expect significant effects on patients' quality of life and considerable lowering in healthcare expenses.
\end{abstract}

\section{Keywords}

Electronic health (E-Health) Remote patient monitoring (RPM), clinical decision support system (CDSS), wireless body area network (WBAN), and electronic health record (EHR)

\section{INTRODUCTION}

Electronic Health (E-Health) refers to the use of electronic communication within health care sector. The technical progress in mobile communications and networking technologies has led to the development of specific systems in e-health labeled as tele-health. Tele-health refers to the remote clinical and non-clinical services (e.g. provide training and medical educations). Tele-medicine is more specific term refers to remote clinical services for patients on remote areas. With the increasing prevalence of mobiles and tablets, it extensively used to support the practice of health care which known as mobile health (m-health). For more clarification. Figure show the relation the relation between e-health systems.

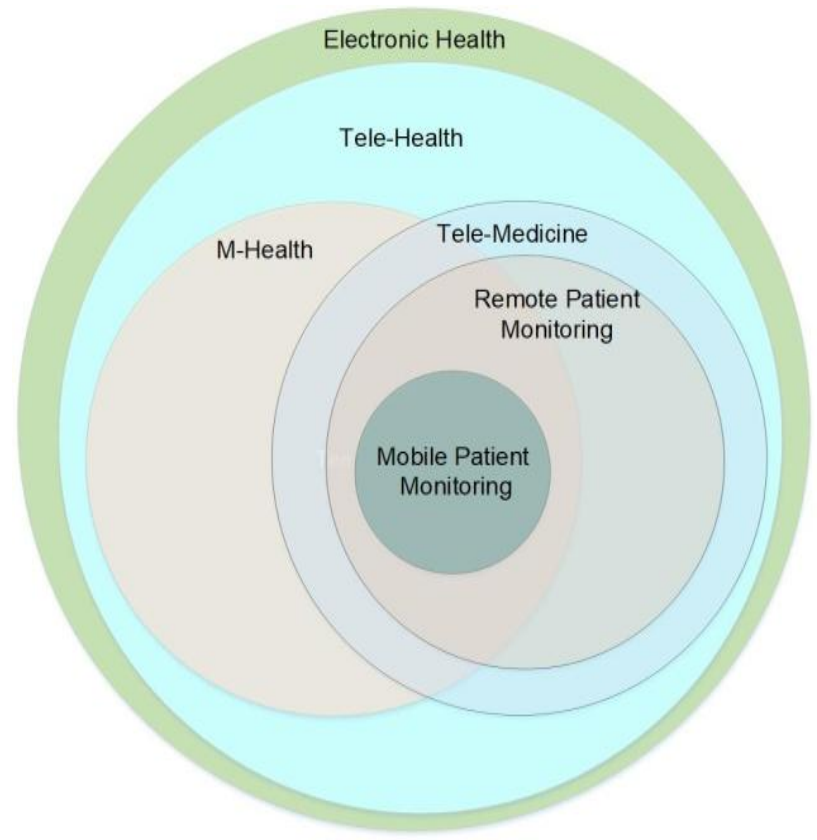

Figure 1: Relations between E- health systems

Developing e-health systems (e.g., remote patient monitoring (RPM), mobile health (m-health), telemedicine, e-visits, econsultations, etc.) are an increasing need. Such systems are used for continuous monitoring, diagnosis, prediction, and treatment. Consequently, they contribute to reducing healthcare costs. Patient monitoring systems considered one of the most important services in mobile health. It helps patients to perform daily activities while their vital signs are fully monitored. Several technologies integrated to provide real time PMs such as Internet of Things (IoT), cloud computing, fog computing clinical decision support systems, etc. Internet of things (IoT) application contribute in enriching business and address various challenges in the health care sector. It facilitates the sensing, processing, and communication of physical and biomedical parameters [1]. The IoT-based wireless body area network (WBAN) is a 
wireless sensor network between wireless devices that enable remote monitoring for different environments Medical environment is one of the promising fields of WBAN for remote, continuous, and real-time patient monitoring using wearable and implantable sensors. S. Ullah et al in [2] surveyed WBAN in medical environments The sensor nodes can be placed either on the patient's body or under his skin to collect certain body parameters or vital signs (e.g., electro cardiogram (ECG), electroencephalogram (EEG), body movement, temperature, blood pressure, blood glucose, heartbeat, and respiration rate levels) The collected data are used for different purposes, such as in-hospital monitoring, remote diagnosis, ambulatory patient monitoring, and trigger emergency services. Every WBAN node has a way for implementation within the body and a role in the network as suggested by IEEE 802.15.6 taxonomy WBAN has a significant role in improving the quality of life, decreasing healthcare costs, and reduction in the workload of medical professions.

Intensive care unit is a critical department in health care sector which admits the most seriously ill patients requiring extensive monitoring (ICU). The need for intensive care units is highly increasing, in response to an aging patient, with the prevalence of chronic diseases and critical patients. Unfortunately, most the Arab states suffer from a serious shortage in hospital rooms as well as ICU beds. As a result, patients whose lives at risk are being turned away from the care unit. Four in five intensive care units are having to send patients to other hospitals due to the shortage of care units [3]. On the other hand, high-risk patients outside hospitals need to be monitored frequently, checked measurements such as temperature, blood pressure, respiratory rate, drip level, etc. Determine the timing of transfer to hospital ICU unit, the admission time to ICU is a critical decision. Various studies demonstrated that, the late identification of clinical deterioration will lead to delay of therapeutic intervention, results in increasing of morbidity and mortality rate [4]. In this paper we propose a complete monitoring system that could monitor patient inside and outside hospitals continuously. The mobile monitoring system will help in this direction, as it could automatically collect patient data, provide an online communication link between patient and healthcare professional wherever he/she is, This increase the healthcare expenditure and improve the quality of patient care.

For our remote monitoring system, we expect to have sensors for fatigue level (EEG), heart rate, respiratory rate, SPO2 saturation, blood pressure, etc. Smart devices, such as phones and tablets, can remotely receive the information sensed by the sensors and act as bridges between patients and doctors, nurses, and pharmacies who take sensitive and real-time decisions depending on the information acquired from those sensors [3]. The patient is free to pursue daily life activities, so this is expected to improve the quality of life. All collected information will aggregated in a distributed EHR repository, which will be stored in the hospital's servers or the on the cloud. The collected real-time data will be used also for making real-time decisions. In order to handle the temporal validity problem, a suitable distributed CDSS system will be embedded as a component in the hospital EHR system [14] and the personal server of the patient's mobile device.

In nutshell, our proposed real-time monitoring for chronic and ICU patient will permit health-care providers to continuously monitor many patients in real time with less effort, provide precise information and continuous communication. It will automatically gather data form WBAN sensors attached on the patient's body, intercommunication network will transform and upload data on the cloud for processing and analyzing. Extracted knowledge will aggregate with Patient EHR on hospital cloud. Distributed CDSS will embed in the distributed EHR that usually in the hospital, to provide realtime monitoring and real-time decision that assist health care providers.

The organization of the paper will be as follows, a literature review is presented in section 2 , and challenges are presented in section 3. Proposed framework and methods are presented in section 4 and 5 separately. Finally, conclusion is presented in section 5

\section{RELATED WORK}

Nowadays, Mobile Health represents important research in health care systems, it aimed at ease a patient's life through telemedicine and mobile health monitoring systems. Remote patient monitoring is widely recognized as an effective way to address current and future challenges of healthcare services, significant efforts carried out to help patients in their daily activities. A comprehensive review of mobile health monitoring system (MHMs) presented by shaker et al [5], and Sruthi et.al [6].Researches in MHMs categorize on three main points (1) patient's data and vital signs acquisition. (2) Data transmission across various network systems. (3) Backend system (database system) that usually located in the hospital. In this literature view, various systems will be analyzed at different levels of details. In [7] authors propose monitoring system for cardiovascular diseases, that use wearable sensors to collect vital signs (Blood pressure, galvanic skin response (GSR), Electrocardiogram (ECG)), authors collect patient vital signs, Bluetooth used to transmit WSN data to mobile device, data stored and classified on patient's mobile, using machine learning algorithm (Support Vector Machine (SVM)), the Extract clinical features used to classify , and determine patient condition "continued risk" or "no longer risk. In[8] Authors propose a mobile monitoring system, that use the noncontact method to automatically measure patient's biomedical signals. The idea of this research is to continuously monitor patient ECG on various locations (office, car, etc.) through fabric sensor electrodes that embedded in a patient chair. Bluetooth used to transmit signals from the sensor to a patient's mobile device. The system provide feedback to the patient when any risk detected, and a web server used to monitor patient health status. Such types of these systems that depend only on mobile device on monitoring process are not suitable for chronic disease patient, as they have many limitations relating to limited storage, reliance on non-complete patient personal and medical data. These limitations prevent their acceptance by the community. Therefore, building effective and efficient RPMs require patient information to be put in a context before making decisions.

Using Cloud service add so much capabilities, that can be divided into three layers of service, Infrastructure as a service (IaaS) which provide high-volume storage such as in Amazon storage service S3, Platform as a service(PaaS), it offers both storage and service such as Google App, and Software as a service (SaaS) . Cloud computing (CC) along with WSN provide promising monitoring systems that enhance QoS. It allows real-time access to patient data at any time and from anywhere, allows preprocessing, analyzing and sharing data, provide high-volume storage in low cost, and reduce the burden in hospitals and clinics. S. Pandey el al in [9] proposed a system that integrates mobile computing and cloud 
computing to analyze ECG data. The authors provided a cloud environment to collect people's health data, such as ECG data, and disseminate them to a cloud-based information repository, which facilitated the analysis of the data using software services hosted in the cloud. N. A. Risso et al in [10] provide Cloud Mobile System (CMS) for monitoring respiratory diseases, system monitor patient Spo2 using wearable sensors, then use mobile device to transmit to it cloud system. In this system authors use cloud as back-end system which permit processing, analyzing, assist patient with treatment. However, system consider non applicable as it depends on Spo2 only to monitor respiratory diseases. P. b Melillo el al in [11] propose monitoring system that used to predict risks of cardiovascular events in hypertensive patients. System use WSN to measure patient's ECG, then use ECG signal to extract Heart Rate Variability (HRV), cloud used as a platform for storage and analyzing of data. A. Rahim el al in [12] authors propose a predictive model that monitor patient vital signs and predict any further anomalous system using Hidden Markov Model (HMM). The same idea In [13] M. M. Rathore el al proposed a real-time CDSS to detect emergence and abnormalities, the system uses wireless sensors to collect patient vital signs, collected data transmitted and stored in the cloud, processing and mining made in a Hadoop ecosystem. In this system, authors focused on format and structure of the heterogeneous collected data, without considering integration between them and between patient EHR, The integration between the heterogeneous collected data, include Patient EHR, wireless sensors, and other unstructured data, requires addressing the challenge of semantic interoperability. Patient EHR provides a complete patient profile of medical data. It collected data from heterogeneous sources such as (different hospital information system, pharmacy information system, laboratory information system, etc.). Each of them has a different standard such as (OpenEHR, HL7, ISO/IEEE 11073, etc.). With different encoding terminology such as (SNOMED CT, LONIC, UMLS)[14][15]. In order to develop applicable RPMs, semantic interoperability must be addressed between sensor-to-sensor, Sensor-to-EHR, between distributed CDSS. In [16], [17] authors provide complete a study about interoperability challenges. many organizations attempt to harmonize data communication and storage, but until now there is no unified standard. For example, ISO has developed IEEE 11073 standard for transmission of blood pressure in binary format between two low-level devices (e.g., sensors), which can be converted to human readable format using HL7 messaging standard (i.e., XML and SNOMED CT). However, HL7 and IEEE 11073 have different coding standards that are cumbersome for low power mobile devices.

For mobile Health solution to be acceptable, applicable, and reliable, it must be standard based. These standards include data standards, security standards, and messaging standards that are combined into a single certifiable solution. Our proposed system is focused on handling challenges of RPMs, the next section will discuss most challenges

\section{THE GENERAL ARCHITECTURE OF RPMS}

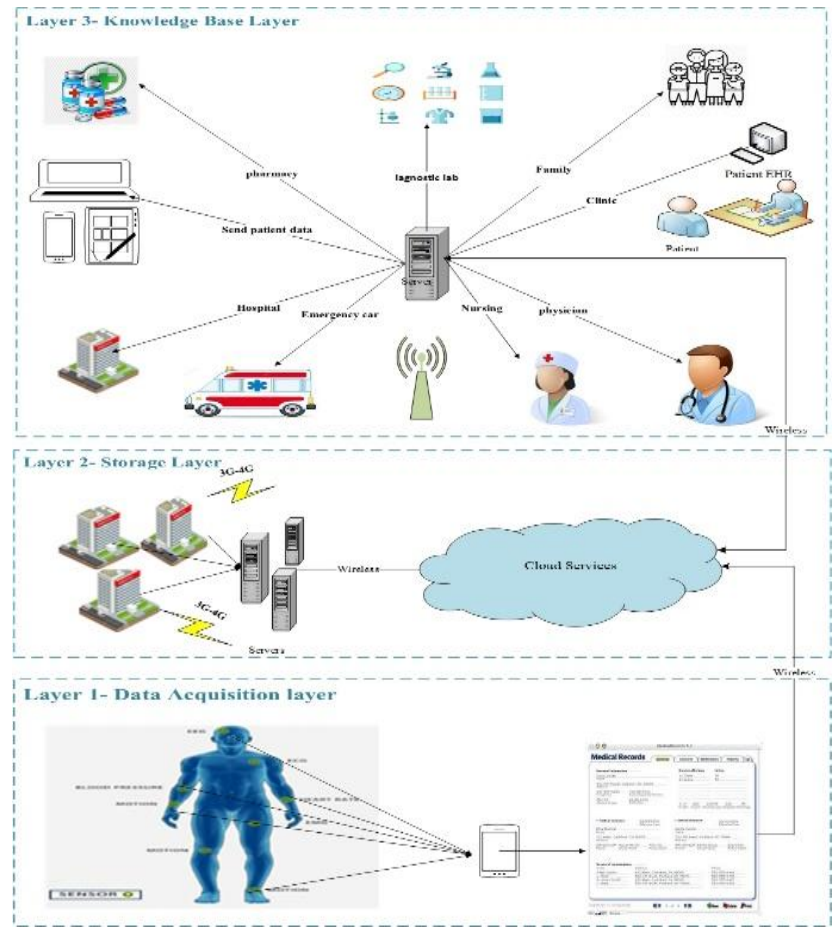

Figure 1: general architecture of RPMs

\subsection{Data Acquisition layer}

Sensors play a key role in a remote monitoring system (RMS). It act as a bridge between the physical world and the digital domain[18]. An RMS uses various sensors to collect data about a patient's health status (i.e., ECG, SPO2, HR, Beat) and context data (i.e. room oxygen level, gas leakage, room temperature, light level, etc.) Actuators can respond to feedback from decision support systems (for example, to adjust insulin dose delivered to a patient based on collected sensors data). A WBAN is an IoT-based wireless network used for remote and real-time monitoring, especially in healthcare applications. A WBAN consists of a set of wearable sensors attached to a patient's body using either invasive or noninvasive techniques [19]. These sensors continuously measure the patient's vital signs and transmit the collected data to a remote location using a wireless communication protocol (i.e., WLAN, WiMAX, LTE, and UMTS). WBA sensor nodes are classified into the following types based on implementation technique:

Implanted Sensors: Sensors implanted either into the patient or under the skin.

External sensors: Sensors directly attached to the patient's skin or separated from the patient by $2-5 \mathrm{~cm}$. A

A WBAN allows RMSs to capture features and information about patients such as their real-time vital signs and supports tracking their symptoms, the effect of their therapy plan, monitoring patient communications, social activities, and even a patient's physiological state. The following points summarize some applications of WBANs in RMSs

- Vital sign monitoring and health assessment systems: realtime patient monitoring and continuous assessment systems are a very useful tool that helps in analyzing patients' health status. It used to monitor patient's activities, predict early risks, and so on. In our study period, several studies were 
concerned with providing PM systems. For example, [20] authors proposed a monitoring system for heart diseases, monitor a patient's vital signs $\left(\mathrm{SpO}_{2}, \mathrm{ECG}\right.$, blood pressure) and then transmit the data according to the patient's health status through four main transmission modes (continuous transmission for all data, continuous transmission at special times, event-triggered transmission and transmission on demand)

- Reminder systems: A reminder system is usually used to help patients with memory impairment like a patient with Alzheimer's. In [21] authors proposed a system that reminded patients with medication dosages at pre-determined times, monitored patient compliance using a Microsoft Kinect and embedded sensors. others [22] used wristband sensors to enable a medication-compliance monitoring system, the idea behind this system was to use a machine-learning algorithm to build and train a classifier to track wrist motion and interference to the schedule for medication adherence. Other medication adherence monitoring and reminder systems were provided in [23][24]

- Fall detection monitoring systems: Falls are a primary cause of injuries, especially for elderly people. In [25], the authors provided a fall detection system based on both wearable and environmental sensors. The concept was authors use a microcontroller unit (MCU) to detect falls based on prior sensor measurements. also [26], the authors proposed a safety alert system that used an accelerometer, cardio meter, and smartphone and constructed a network between these sensors via Bluetooth. When any abnormal sensor readings occurred, it automatically informed health care professionals and the family and identified the patient and their location

\subsubsection{Challenges related to WBAN and RPMs}

Challenges of dealing with WBAN are common is most medical do domains, the patient in RPMs needs to be monitored with wireless sensors that collect patient vital signs such as ECG, heart rate, SPO2, blood pressure, blood glucose. Following are summarize of challenges in this area

- The power consumption of WSN is a big challenge for RPMs, usually, the capacity of batteries consumes in sensing, processing, and transmission so that it requires frequent recharging, and it may consider the weak point in RPMs, especially; in case of patients is unable to monitor batteries and frequently charge it [27].

- Design of WSN should also consider sensor weight, patient mobility, network coverage. A homogenous WSN provide an advantage in network coverage and energy consumption but still has limitations in power consumption and in number of patients which connect to one sink [28].

- The accuracy of Sensors in sensing still not reached a stable state, therefore various challenges work on enhancing signal processing and transmission.

- Not all smart devices support the automatic transmission of patient data without human intervention.

- Communication standards between devices (invasive-non-invasive) still not reach to stable status, as each standard developed for specific device, for example; IEEE 802.15.6 developed for WBAN sensors, however, is considered not suitable for some cases [29], others like ANT, WIFI,
ZigBee and Bluetooth have many routing problems in WBAN, each of them provide different QoS related to power consumption, security and reliability

\subsection{Storage layer}

\subsubsection{Cloud computing}

Cloud computing is a new paradigm that adds fruitful benefits to healthcare care systems. It can be used by both hardware and software systems to deliver data over the Internet. It helps to process, analyze, sort and transmit data securely, etc. [30][31]. Benefits could be summarized at the following points.

- Increase speed and efficiency: Cloud monitoring systems usually use a group of servers to manage patient status, which increases system speed and efficacy. In [32] authors showed that cloud computing increased the speed by 20 times and the energy efficiency by 10 times. Also, a hardware failure in one server does not dramatically affect the performance of the overall system.

- Big data storage: it provides enormous amounts of storage for saving large healthcare data files (e.g., imaging data, X-ray images, etc.). Besides, it provides a means to share these files easily with other hospitals.

- Healthcare analytics: it helps in analyzing all patients' data (such as demographics, symptoms, therapy plans and treatment) and makes all the data available to support and improve clinical decisionmaking processes and medical research.

- Data security: Cloud computing provides more secure servers than do local servers for storing patient data. Clouds use security layers to secure cloud data from theft or hacks.

Cloud computing in conjunction with a WSN enables promising monitoring systems that can enhance the quality of service (QoS). The combination offers physicians the ability to monitor all patient data sensed with biosensors regardless of the type of patient data, it contributes to reducing the burden of hospitals and clinics. To take full advantage of, several challenges and limitations should be handled. Limitations and challenges of cloud computing in PMs are confined to the power and time consumption, privacy and security of the transmitted data. Several systems have been proposed to address these issues. First; Some studies have focused on increasing the transmission speed by providing routes with low end-to-end delay, as in[30], a cloud health monitoring system (CHMS) was proposed to track nonhospitalization patients and improve QoS. authors in this work try to address many challenges. First, it overcomes the delay in the delivery time of urgent data. They classified patient data as urgent and non-urgent at different frequencies. The urgent data was immediately transmitted to the medical staff, while lower-frequency data was aggregated and transmitted at a low frequency. Second, they worked on the mutual interference problem by using a dynamic channel policy that distributed WBANs to the available channel. This system also switched and selected the available channel using the master node concept. Another Systems have been proposed to schedule and prioritize the transmission of vital signs over multiple channels based on the patient's current status [33]. In this system [34], the author's categorized patients into three 
classes (red, green and blue) that clarified the level of the required treatment (e.g., immediate, delayed, or minimal). Other studies focused on energy and time consumption in mobile-cloud monitoring systems. Since battery constraints are considered one of the main challenges in mobile monitoring systems. The authors [32] proposed a system that suggests running some parts of the application components in parallel which helps save batteries and avoids draining too quickly.

\subsubsection{Fog computing}

Fog is "cloud close to the ground", it extends the traditional cloud to edge network. The point of using fog computing is to transfer the processing of some sensitive application to the edge (near the end device), while others cane done over the cloud. Problems related to location awareness, reliability, latency and many other challenges are resolved by fog computing [35]. The fog computing in PM systems in a new concept in this domain. It provides many advantages over cloud, it summarized in the following points:

- In fog computing, data processed and analyzed locally instead of sending it to the cloud, this led to less amount of bandwidth consuming, decrease the overall cost[36].

- Processing data locally will decrease the timelatency during transmission which help to avoid problems especially for time-sensitive application (e.g. real-time monitoring, self-driving car, etc..).

- Providing better privacy to users, as patient's data can be analyzed locally instead of sending it to the cloud

- Deploying fog servers in PMs decrease the required bandwidth for transmission, providing real-time data to doctors without the need of internet connection[37].

- Utilizing fog computing will not only help people to get ease on their basic health monitoring, but will also help those countries where there is less doctor to patient ratio[38].

- It also provides additional advantages such as save power consumption while continuous transmitting to cloud servers[39].

Fog IoT systems divided into three main layers include (device layer, fog layer and cloud layer) instead of (device layer, cloud layer) in cloud computing systems. In [40] authors provide a tutorial that discuss the differences between edge computing, fog computing and cloud computing, in addition to the advantages of using fog computing. Others survey[41][38] have been discussed the role of fog computing in various domains. Fig 14 show the basic of fog computing model for PMs.

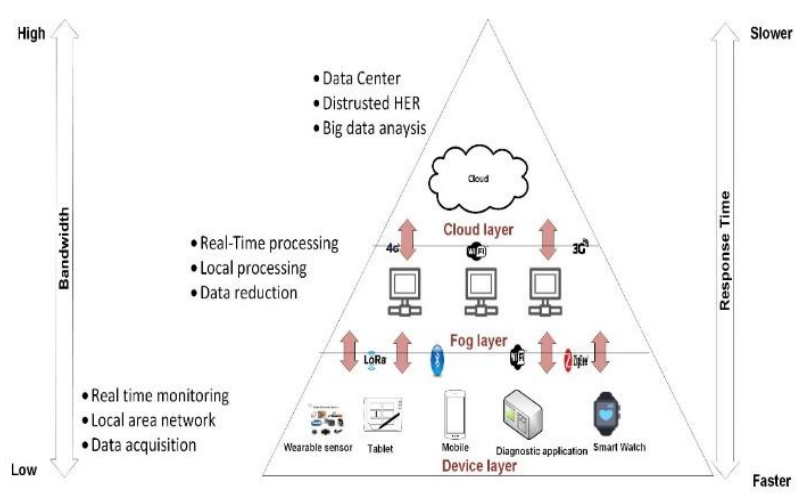

Figure 14: Fog computing in Remote monitoring system

In [37] authors provide smart health monitoring system based on fog nodes. In this system, edge users are equipped with various kinds of wearable sensors and devices used to aggregate medical measurements. these devices can have different connectivity such as Bluetooth, Zigbee, LoRa, etc. it used LoRaWAN to send aggregated measurements directly to the closer fog nodes or health centers. Authors use LoRAWAN as it could transmit across one ten kilometers even if there is no internet connection. This will not only help patients with continuous monitoring regardless of internet connection but also decrease the overall bandwidth, the battery consumption. Others in [42] provide low-cost monitoring system of ECG and temperature. Authors use sensor node to collect data and wireless transmit to the nearest gateway accessed by caregivers. It provides real-time monitoring and help in decision making process. The same in [43], they exploiting the fog computing concept to provide enhancing health monitoring system. They depend on the smart gateway in analysis of ECG, extract the critical features such s P and T waves. Achieving bandwidth efficiency and decrease latency. In [44], authors provide system to control and prevent Zika virus. They used fog computing as an intermediary layer between users and the cloud server to decrease the latency and cost of communication in such high cloud-based systems. Table show differences between fog and cloud computing.

Cloud computing and fog computing have their advantages and disadvantages. In many real cases, they need to cooperate with each other. Cloud nodes provide global centralization, while fog nodes provide localization. For more clarification about the difference between cloud computing and fog computing, table 1 provide in terms of many factors.

Table 1: Difference between cloud computing and fog computing

\begin{tabular}{|ccc|}
\hline factor & Cloud computing & Fog computing \\
\hline latency & High & low \\
mobility & limited & supported \\
Geo-distribution & centralized & distributed \\
Bandwidth cost & High & Low \\
Storage capability & strong & weak \\
Energy & high & low \\
consumption & & \\
Location & Partially & Fully supported \\
awareness & supported & \\
\hline
\end{tabular}




\begin{tabular}{|c|c|c|}
\hline No. of server & few & large \\
\hline $\begin{array}{l}\text { Real-time } \\
\text { interaction }\end{array}$ & supported & supported \\
\hline security & undefined & defined \\
\hline $\begin{array}{l}\text { Location of } \\
\text { service }\end{array}$ & With the internet & $\begin{array}{l}\text { At the edge of the } \\
\text { local network }\end{array}$ \\
\hline
\end{tabular}

\subsection{Knowledge base layer}

This section will focus on the knowledge base layer (third layer), and its role in remote patient monitoring. A clinical decision support system (CDSS) is considered to be the brain of a healthcare system, it used to assist healthcare teams in the decision-making process [45]. The literature indicates that the use of CDSSs has an important impact on monitoring systems. CDSSs may include many functions, described as follows. (I) It provides a comprehensive health care view of the patient's medical history. (II) Helps non-expert physicians by providing clinical guidelines, practice standards, and differential diagnoses. (III) help a patient by offering several assistive tools such as drug-schedule reminders, drug prescriptions, drug doses, drug alternatives and interactions with other drugs, devices and recommendations based on patient EHRs and knowledge bases (KB)

All these factors improve the efficiency and reliability of using DSS in remote monitoring systems especially in remote areas. To build efficient DSS various component must be integrated to provide complete image about patient health status such as (patient EHR, Knowledge base, clinical principle guidelines, etc.). unfortunately, integrating between these components have many challenges related to size and type of data. These challenges could be summarized in the following subsections.

\subsubsection{Challenges related to big data and RPMs}

Most of the medical application based on remote monitoring, treat with a large volume of data include medical and WBAN sensor data. Besides that, most medical data are often unstructured and include data from so many resources. The big data technology is very suitable for these types of environments, but it has so many challenges.

- Capturing, storing, and analyzing of real-time medical data is considered a challenge for local hosting, Therefore, using cloud computing provide a better environment to deal with these challenges. However, the network transmission speed is a bottleneck [46].

- Semantic interoperability between different network layers and different devices (i.e. sensors, smart devices, and cloud servers), and between various structured data, semi-structured such as (XML, CSV, and JSON) is a big issue that needs to be handled [47].

- Medical data for RPMs usually have special nature, as it is huge in volume, capture form many resources such as (relational database, XML documents, CSV files, etc.), contain complex, incomplete, noisy uncertain data [48]. Therefore, traditional machine learning techniques are not suitable, intelligent ML algorithms should be used to deal with semantic and temporal data and extract information.
- Results of Machine learning (ML) continuously refresh decision support system knowledge base, therefore parallel processing and portioning should be used to convert CDSS to a predictive tool that can predict patient status in the near future

- A proportion of medical data is usually unstructured data, but it contains valuable information. Using standard ontologies can handle this issue by improving representation, integration, meaning to extract information[49]

\subsubsection{Challenges related to CDSS and RPMS}

Remote patient monitoring systems must have CDSS that work both on patient side and hospital side, therefore CDSS must consider patient EHR, WBAN real-time data, and up-todate domain expert, in order to give real-time advice and decisions. Following are some challenges that need to be handled to build distributed, accurate CDSS

- CDSS must depend on patient complete patient profile, to provide an accurate decision for each patient, some of the current researches provide high accuracy decisions [50], but they depended only on a small number of features, therefore; such types of CDSS are not acceptable for most healthcare professionals

- $\quad$ CDSS give a prediction based on real-time patient's data., As a result, using temporal data mining algorithms is essential to mine information, and refresh CDSS knowledge base to make to it up-todate [51].

- $\quad$ CDSS have to integrate data from multiple sources include (Patient EHR data, domain experts, CPGs, etc.) in distributed CDSS that have different structure and format, as a result, it considers challenge to mine across various heterogenous data[52].

- Mostly RPMs developed to help disabled and elderly people, therefore; it's important to develop an intelligent interface that utilizes Brain-Computer Interface (BCI) and Human-computer interaction (HCI) which support dynamically creation of user interface, and automatic gathering of patient personal data such as patient's feeling, mood and symptoms [53]

\subsubsection{Challenges related to EHR and RPMs}

Chronic diseases cannot be managed without patient complete EHR, An EHR contains comprehensive patient's medical data, the EHR ecosystems collected from different sources include, laboratory information system, Intensive care unit, radiology information. All of these systems must communicate and work as a single unit, following are some challenges related to EHR.

- Current EHR systems have different standards (e.g., HL7, OpenEHR, ASTM, CCD, ISO /IEEE 11073) and different terminology encoding systems (e.g. LONIC, SCT, ICF, CPT 4, ATC, UMLS), but until this time there is no unified standard for data communication, integration, and storage, Therefore, maintaining Syntax and semantic interoperability among different systems is a big challenge [5].

- Centralized and distributed middleware can provide solution by offering gateway between systems, but 
it still have many limitations [54], [55] [56].

- RPMs that based on WSN data add additional complexity, by adding interoperability challenge, to process and integrate the big data extracted WBAN sensors with patient HER in real time.

- Generally, Interoperability consider big challenge mobile health application, especially in RPMs, as it should deal and solve the problems related to sensor-to-sensor interoperability, PHR to distributed EHR integration, distributed EHR to WBAN integration, the CDSS to EHR integration, and CDSS to CDSS integration and it consider a complex issue

\section{PROPOSED FRAMEWORK}

As discussed in related work section, they are many RPM systems, but most of them focus in help patients by monitoring vital signs through wearable sensors, and other focus on other points include (data security, transmission and integration). None of the previous researches propose end-to end solution. Therefore, a complete RPMs proposed in this research. System will help in monitoring patient, predict future health status, prevent risks and complications. As shown in figure 1 , framework divided into 3 stages. The patient side, the cloud side, the back-end server side. Framework also contain 3 components for DSS, it used to help patients and physician. The next section discusses framework modules in details

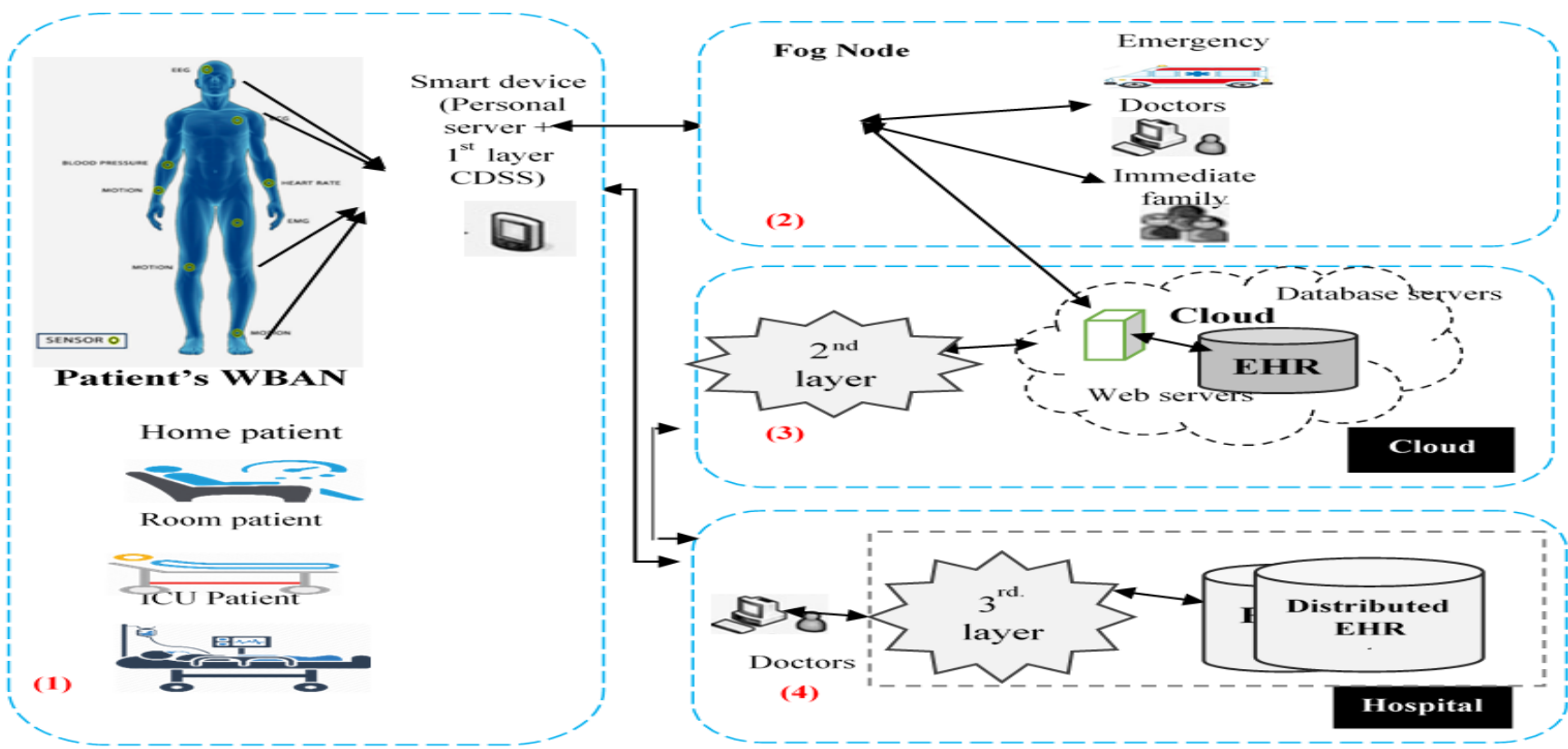

Figure 1: Mobile Health monitoring Framework

\subsection{Patient Side}

The Patient side is fed by vital signs captured from various sensors, attached on patient's body and collected on central control unit. One of the barriers on using WSN is missing data due to patient's body movements. Therefore, major $\mathrm{MH}$ monitoring applications reported that the accuracy are affected by missing and noisy data [20]. Most noises cannot be filtered by hardware processing unit. Therefore, it is important to filter out dataset as much as possible, various filters such as band-pass filter, finite impulse response (FIR) to decrease noise and motion artifacts. After processing data, it transferred to patient smart device using Bluetooth. Another common issue that affect results of $\mathrm{MH}$ systems with $W S N$, is the delay in transmission due to signal strength, transmission speed, etc. To handle this issue, in our proposed system data transmitted in two modes (online mode and offline mode), accordance to patient vital signs (regular data, urgent data) as shown in figure 2

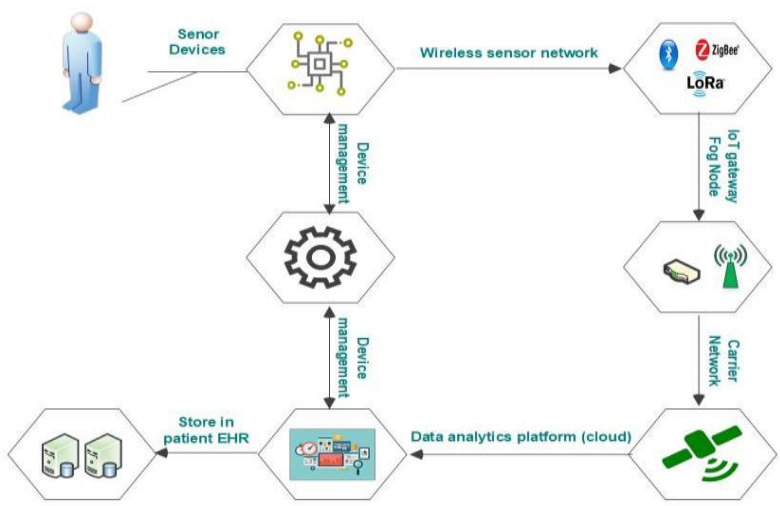

Figure 2: patient's data transmission mode

Wireless sensors will be deployed in patient's home to monitor patient vital signs such as temperature, SPO2, blood pressure, etc. The collected data from sensors then will send to fog cloud server using LoRa network (most near hospital). Data in each fog node will continuously updated by transmitting data to cloud nodes using standard communications (MQTT and HTTP). The framework aslo includes a component for intelligent CDSS systems at three level patient's side, Cloud Side and service provider's side. CDSS in patient's side (i.e., $1^{\text {st }}$ layer CDSS) is a light system with embedded machine learning algorithms. It used to 
monitor patient health status, provide time sensitive and fast decision that doesn't depend on internet connection and don't consume battery life. It uses data collected form from WSN to define alerts, reminders, drug dosing, clinical risks and drug to drug allergies and other decisions that doesn't need high processing. In case patient at risk, system immediate send SMS to physician

As we believe that one of the main advantages of mobile health monitoring systems is the patient's self-management of his her health condition and treatment. Therefore, our $\mathrm{MH}$ monitoring system in patient's side designs to be not only data collector toll, but also to be self-motivating and intelligent interface that utilizes Human-computer interaction (HCI). It supports the dynamically creation of user interface, according to patient personal data such as patient's feeling, mood and symptoms.

\subsection{Fog \& Cloud computing}

$\mathrm{CC}$ is the core of MHM systems. Wireless sensors and mobile devices automatically collect patient data (e.g., vital signs, personal data, EHRs). Continuously collecting these data generates a large amount of information that is not commensurate with the potential of mobile devices (limited memory and processing power) [5] Most of health care application using sensor-to cloud architecture, which is not feasible. Using a cloud environment provides nearly unlimited storage for real-time patient data. But on the other hand, relying on remote data centers in all data analysis in not acceptable because of network failure and missing data. In addition to that, continuous transmission of patient's data will cause battery consumption. Hence the need for fog computing .Using fog computing aims to improve system performance and efficiency [36], it reduce cloud involvement, by doing most data analysis and pre-processing steps in IOT devices connected to fog computing environment [57], fog cells are software running on IOT devices (i.e. gateways, access points, routers) every fog cell or fog node connected to fog control node, if one the fog cell failed to receive data it will automatically connect fog control point or to fog-cloud middle ware. Using fog computing provide many advantages over cloud computing, especially in healthcare side which can be summarized as follows (1) reduce volume of data transmitted to cloud, as it provides filter, pre-process, and remove raw data locally. (2) provide analyzing for sensitive data locally which increase privacy. (3) save battery as it transmits data to cloud after fixed period separated sleep cycle. During sleep cycle, gateway tack care of any risks or requests

Briefly, Transmission of patient's data will divide in two modes (Nowcasting \& Forecasting) based on patient status collected in $1^{\text {st }}$ CDSS. If patient classified as high-risk data, patient's data will send to cloud in real time. Otherwise, it locally sends to fog nodes and transmit every fixed period to cloud servers

\subsection{Service provider}

In hospital side, consider the back-end system of $\mathrm{MH}$ monitoring system. It contains distributed EHR, medical expert, and knowledge base. Patient's EHR contains patient's historical medical data, patient current data which include various data and structure. All patient's EHR aggregated on local hospital EHR. Each hospital has local EHR, local medical expert for a specific domain that continuously changed by adding real-time data. Analyzing these data takes long time; therefore, an automated CDSSs is highly required to maximize the benefits of using EHR.CDSS in back end system (CDSS 3rd layer) depends on the whole distributed EHR, up-to-date knowledge base. CDSS knowledge base must depend on up-to-date data extracted form Clinical Practice Guidelines (CPGs) and machine learning and doctor's opinions, To maximize the benefits of using CDSS, data mining will continuously work on every local knowledge base and EHR to ensure that all EHRs are updated. Extracted knowledge added to the knowledge base that used in building applicable These CDSS systems will use fuzzy and semantic ontologies technologies to enhance their semantic and intelligent capabilities in hospital.

\section{CONCLUSION AND FUTURE WORK}

Chronic diseases are considered the major reason that cause death. MH monitoring systems consider efficient solutions that provide scalable and affordable solutions. In this paper, we proposed $\mathrm{MH}$ monitoring framework to monitor chronic diseases patient and ICU patient. System concentrate on two main points (1) monitor patient at any time and form and anywhere, (2) predict patient vital signs in the near future. Integrating WSN with $\mathrm{CC}$ provide promising environment to overcome MHMs limitations Our main goal with this work is to provide a complete framework for a chronic diseases patients, reduce the number of regular visits to the hospital, provide continuous monitoring that avoids life-threatening complications, Improve communications between patients and their relatives, and care providers. Overall, we aim to easy patient life and increase the quality of life. In order to make framework applicable, scalable and acceptable, privacy security of patient data and resource-constraint should take into account Our proposed framework will be implemented and tested in many scenarios such as monitoring patients everywhere and every time, monitoring patients in-hospital and monitoring patients in emergency situations. Further work, will implement and experiment every component of framework, propose secure protocol to ensure data security

1), use no additional space above the subsection head.

\section{REFERENCES}

[1] G. Elhayatmy, N. Dey, and A. S. Ashour, Internet of Things Based Wireless Body Area Network in Healthcare. 2018.

[2] S. Ullah, P. Khan, N. Ullah, S. Saleem, H. Higgins, and K. S. Kwak, "A Review of Wireless Body Area Networks for Medical Applications," Int. J. Commun. Netw. Syst. Sci. (IJCNS), vol. 2, no. 8, pp. 797-803, 2009.

[3] "ICU Shortage." [Online]. Available: https://www.theguardian.com/society/2018/mar/07/patie nts-turned-away-intensive-care-lack-beds-shortagehospitals. [Accessed: 19-Nov-2018].

[4] J. Yoon, A. Alaa, S. Hu, and M. Schaar, "ForecastICU: A Prognostic Decision Support System for Timely Prediction of Intensive Care Unit Admission," Proc. 33rd Int. Conf. Mach. Learn., vol. 48, pp. 1680-1689, 2016.

[5] S. El-Sappagh, F. Ali, S. El-Masri, K. Kim, A. Ali, and K.-S. Kwak, "Mobile Health Technologies for Diabetes Mellitus: Current State and Future Challenges," IEEE Access, vol. PP, no. c, pp. 1-1, 2018.

[6] K. Sruthi, E. V Kripesh, and K. A. U. Menon, "A survey of remote patient monitoring systems for the measurement of multiple physiological parameters," pp. 153-159, 2017. 
[7] O. Boursalie, R. Samavi, and T. E. Doyle, "M4CVD: Mobile Machine Learning Model for Monitoring Cardiovascular Disease," Procedia - Procedia Comput. Sci., vol. 63, no. Icth, pp. 384-391, 2015.

[8] E. M. Fong and W. Y. Chung, "Mobile cloudcomputing-based healthcare service by Noncontact ECG monitoring," Sensors (Switzerland), vol. 13, no. 12, pp. 16451-16473, 2013.

[9] S. Pandey, W. Voorsluys, S. Niu, A. Khandoker, and R. Buyya, "An autonomic cloud environment for hosting ECG data analysis services," Futur. Gener. Comput. Syst., vol. 28, no. 1, pp. 147-154, 2012.

[10] N. A. Risso et al., "A cloud-based mobile system to improve respiratory therapy services at home," J. Biomed. Inform., vol. 63, pp. 45-53, 2016.

[11] P. . b Melillo, P. . Scala, F. . Crispino, and L. . c Pecchia, "Cloud-based remote processing and data-mining platform for automatic risk assessment in hypertensive patients," Lect. Notes Comput. Sci. (including Subser. Lect. Notes Artif. Intell. Lect. Notes Bioinformatics), vol. 8868, pp. 155-162, 2014.

[12] A. Rahim, M. Forkan, and I. Khalil, "A Probabilistic model for early prediction of abnormal clinical events using vital sign correlations in home-based monitoring," 2016.

[13] M. M. Rathore, A. Ahmad, A. Paul, J. Wan, and D. Zhang, "Real-time Medical Emergency Response System: Exploiting IoT and Big Data for Public Health," J. Med. Syst., vol. 40, 2016.

[14] S. Riad and M. Zaghloul, "A Preparation Framework for EHR Data to Construct CBR Case-Base," Handb. Res., no. April, 2017.

[15] U. Varshney, "Pervasive healthcare computing: EMR/EHR, wireless and health monitoring," Pervasive Healthc. Comput. EMR/EHR, Wirel. Heal. Monit., pp. $1-282,2009$.

[16] M. Sotoodeh, "Challenges in Semantic Interoperability in Emergency Management," no. 2.

[17] S. Pileggi and C. Fernandez-Llatas, Semantic Interoperability: Issues, Solutions, Challenges. 2012.

[18] P. K. Sahoo, H. K. Thakkar, and M. Y. Lee, "A cardiac early warning system with multi channel SCG and ECG monitoring for mobile health," Sensors (Switzerland), vol. 17, no. 4, pp. 1-28, 2017

[19] Y. E. Gelogo, H. J. Hwang, and H. Kim, "Internet of Things ( IoT ) Framework for u-healthcare System," vol. 9, no. 11, pp. 323-330, 2015.

[20] C. Li, X. Hu, and L. Zhang, "The IoT-based heart disease monitoring system for pervasive healthcare service," in Procedia Computer Science, 2017, vol. 112, pp. 23282334.

[21] X. Wang et al., "Eye Contact Reminder System for People with Autism," Proc. 6th Int. Conf. Mob. Comput. Appl. Serv., 2014.

[22] V. Moshnyaga, M. Koyanagi, F. Hirayama, A. Takahama, and K. Hashimoto, "A medication adherence monitoring system for people with dementia," 2016 IEEE Int. Conf. Syst. Man, Cybern. SMC 2016 - Conf.
Proc., no. 165008, pp. 194-199, 2017.

[23] V. J. Silva, M. A. S. Rodrigues, R. Barreto, and V. F. De Lucena, "UbMed: A ubiquitous system for monitoring medication adherence," 2016 IEEE 18th Int. Conf. eHealth Networking, Appl. Serv. Heal. 2016, pp. 16-19, 2016.

[24] N. Hezarjaribi, R. Fallahzadeh, and H. Ghasemzadeh, "A machine learning approach for medication adherence monitoring using body-worn sensors," 2016 Des. Autom. Test Eur. Conf. Exhib., pp. 842-845, 2016.

[25] J. Wang, Z. Zhang, B. Li, S. Lee, and R. Sherratt, "An enhanced fall detection system for elderly person monitoring using consumer home networks," IEEE Trans. Consum. Electron., vol. 60, no. 1, pp. 23-29, 2014.

[26] N. R. Singh, P. R. Rothe, and A. P. Rathkanthiwar, "Implementation of safety alert system for elderly people using multi-sensors," Proc. Int. Conf. Electron. Commun. Aerosp. Technol. ICECA 2017, vol. 2017-Janua, pp. 282-286, 2017.

[27] N. Kalid, A. A. Zaidan, B. B. Zaidan, O. H. Salman, M Hashim, and H. Muzammil, "Based Real Time Remote Health Monitoring Systems: A Review on Patients Prioritization and Related 'Big Data' Using Body Sensors information and Communication Technology,' J. Med. Syst., vol. 42, no. 2, 2018

[28] O. Salem, Y. Liu, A. Mehaoua, and R. Boutaba, "Online Anomaly Detection in Wireless Body Area Networks for Reliable Healthcare Monitoring," IEEE J. Biomed. Heal. Informatics, vol. 18, no. 5, pp. 1541-1551, 2014.

[29] G. Yang et al., "IoT-based Remote Pain Monitoring System: from Device to Cloud Platform," IEEE J. Biomed. Heal. Informatics, vol. 2194, no. c, 2017.

[30] G. Almashaqbeh, T. Hayajneh, A. V. Vasilakos, and B. J. Mohd, "QoS-Aware Health Monitoring System Using Cloud-Based WBANs," J. Med. Syst., vol. 38, no. 10 , 2014.

[31] "Advantages of Cloud Monitoring of Environmental Conditions," 2015

[32] J. H. Ahnn and M. Potkonjak, "mHealthMon: Toward Energy-Efficient and Distributed Mobile Health Monitoring Using Parallel Offloading," J Med Syst, vol. 37, pp. 1-5, 2013.

[33] G. Almashaqbeh, "A Cloud-based Interference-aware Remote Health Monitoring System for Non-hospitalized Patients," 2014, pp. 2436-2441.

[34] B. Chen, J. P. Varkey, D. Pompili, J. K.-J. Li, and I. Marsic, "Patient vital signs monitoring using Wireless Body Area Networks," Proc. 2014 IEEE 36th Annu. Northeast Bioeng. Conf., pp. 1-2, 2010.

[35] P. Verma and S. K. Sood, "Fog Assisted- IoT Enabled Patient Health Monitoring in Smart Homes," no. February, 2018.

[36] S. Yi, C. Li, and Q. Li, "A Survey of Fog Computing," Proc. 2015 Work. Mob. Big Data - Mobidata '15, no. August, pp. 37-42, 2015.

[37] J. Kharel, H. T. Reda, and S. Y. Shin, "An architecture for smart health monitoring system based on fog 
computing," J. Commun., vol. 12, no. 4, pp. 228-233, 2017.

[38] [38] I. Stojmenovic, "Fog computing: A cloud to the ground support for smart things and machine-to-machine networks," in 2014 Australasian Telecommunication Networks and Applications Conference (ATNAC), 2014, pp. 117-122.

[39] F. A. Kraemer, A. E. Braten, N. Tamkittikhun, and D. Palma, "Fog Computing in Healthcare - A Review and Discussion," IEEE Access, vol. 5, pp. 9206-9222, 2020.“fogsurevy.".

[40] A. V Dastjerdi and R. Buyya, "Fog Computing: Helping the Internet of Things Realize Its Potential," Computer (Long. Beach. Calif)., vol. 49, no. 8, pp. 112-116, Aug. 2016.

[41] T. Nguyen Gia et al., "Low-cost fog-assisted health-care IoT system with energy-efficient sensor nodes," 2017 13th Int. Wirel. Commun. Mob. Comput. Conf. IWCMC 2017, pp. 1765-1770, 2017.

[42] T. N. Gia, M. Jiang, A. Rahmani, T. Westerlund, P. Liljeberg, and $\mathrm{H}$. Tenhunen, "Fog Computing in Healthcare Internet of Things: A Case Study on ECG Feature Extraction," in 2015 IEEE International Conference on Computer and Information Technology; Ubiquitous Computing and Communications; Dependable, Autonomic and Secure Computing; Pervasive Intelligence and Computing, 2015, pp. 356363.

[43] S. Sareen, S. K. Gupta, and S. K. Sood, "An intelligent and secure system for predicting and preventing Zika virus outbreak using Fog computing," Enterp. Inf. Syst., vol. 11, no. 9, pp. 1436-1456, Oct. 2017.

[44] P. K. Anooj, "Clinical decision support system: Risk level prediction of heart disease using weighted fuzzy rules," J. King Saud Univ. - Comput. Inf. Sci., vol. 24, no. 1, pp. 27-40, 2012.

[45] M. Jung, K. Han, and J. Cho, "Advanced verification on WBAN and cloud computing for u-health environment," Multimed. Tools Appl., vol. 74, no. 16, pp. 6151-6168, 2015.

[46] A. Bahga and V. K. Madisetti, "A cloud-based approach for interoperable electronic health records (EHRs)," IEEE J. Biomed. Heal. Informatics, vol. 17, no. 5, pp. 894-906, 2013.

[47] Z. Marzuki and F. Ahmad, "Data Mining Discretization Methods and Performances," Mach. Learn., no. 1, pp. 978-980, 2007.

[48] A. Wul, B. Haarbrandt, E. Tute, M. Marschollek, P. Beerbaum, and T. Jack, "Arti fi cial Intelligence In Medicine An interoperable clinical decision-support system for early detection of SIRS in pediatric intensive care using openEHR," Artif. Intell. Med., no. April, pp. $0-1,2018$.

[49] V. F., C. L., R. J., B. F., G. J.B., and M. N., "Clinical decision support systems (CDSS) for preventive management of COPD patients," J. Transl. Med., vol. 12, no. Suppl 2, p. no pagination, 2014.

[50] S. Shanthi and D. Kumar, "Prediction of blood glucose concentration ahead of time with feature based neural network," Malaysian J. Comput. Sci., vol. 25, no. 3, pp. 136-148, 2012.

[51] A. M. Alaa, K. H. Moon, W. Hsu, and M. van der Schaar, "ConfidentCare: A Clinical Decision Support System for Personalized Breast Cancer Screening," vol. XX, no. X, pp. 1-13, 2016.

[52] A. I. N. Press, "Human - Computer Interaction for Medicine and Health Care ( HCI4MED ): Towards making Information usable," vol. 68, pp. 325-327, 2010.

[53] R. K. Lomotey and R. Deters, "Efficient mobile services consumption in mHealth," in 2013 IEEE/ACM International Conference on Advances in Social Networks Analysis and Mining (ASONAM 2013), 2013, pp. $982-989$.

[54] W. A. Khan et al., "An Adaptive Semantic based Mediation System for Data Interoperability among Health Information Systems," J. Med. Syst., vol. 38, no. 8, p. 28, Jun. 2014.

[55] S. El-Sappagh and F. Ali, "DDO: a diabetes mellitus diagnosis ontology," Appl. Informatics, vol. 3, no. 1, p. 5,2016 .

[56] P. Hu, S. Dhelim, H. Ning, and T. Qiu, "Survey on fog computing: architecture, key technologies, applications and open issues," J. Netw. Comput. Appl., vol. 98, pp. 27-42, 2017.

[57] K. M. D. M. Karunarathna, "Predicting ICU death with summarized patient data," 2018 IEEE 8th Annu. Comput. Commun. Work. Conf. CCWC 2018, vol. 2018Janua, pp. 238-247, 2018.

[58] T. Desautels et al., "Prediction of early unplanned intensive care unit readmission in a UK tertiary care hospital: A cross-sectional machine learning approach," BMJ Open, vol. 7, no. 9, pp. 1-9, 2017.

[59] S. M. Vieira, J. P. Carvalho, A. S. Fialho, S. R. Reti, S. N. Finkelstein, and J. M. C. Sousa, "A decision support system for ICU readmissions prevention," Proc. $2013 \mathrm{Jt}$. IFSA World Congr. NAFIPS Annu. Meet. IFSA/NAFIPS 2013, pp. 251-256, 2013.

[60] A. P. Kurniati, G. Hall, D. Hogg, and O. Johnson, "Process mining in oncology using the MIMIC-III dataset,” J. Phys. Conf. Ser., vol. 971, no. 1, 2018. 